\title{
La gestion des connaissances dans les organisations
} québécoises : état des lieux, enjeux actuels, avenir Knowledge Management in Quebec Organizations: State of Affairs, Current Issues, Future La gestión del conocimiento en las organizaciones quebequenses: estado actual, retos y perspectivas para el futuro

\author{
Kimiz Dalkir
}

Volume 54, numéro 2, avril-juin 2008

Topographie du Québec documentaire

URI : https://id.erudit.org/iderudit/1029323ar

DOI : https://doi.org/10.7202/1029323ar

Aller au sommaire du numéro

Éditeur(s)

Association pour l'avancement des sciences et des techniques de la documentation (ASTED)

ISSN

0315-2340 (imprimé)

2291-8949 (numérique)

Découvrir la revue

Citer cet article

Dalkir, K. (2008). La gestion des connaissances dans les organisations québécoises : état des lieux, enjeux actuels, avenir. Documentation et bibliothèques, 54(2), 139-142. https://doi.org/10.7202/1029323ar
Résumé de l'article

L'auteure passe en revue la gestion des connaissances (GC) au Québec dans trois secteurs primaires : les grandes universités québécoises, le secteur public provincial et certaines grandes organisations du secteur privé. Une bonne partie des projets de GC implique un partenariat entre ces trois secteurs. Cependant, la GC au Québec souffre encore d'un manque de visibilité nationale et internationale. L'article ne propose pas un inventaire exhaustif puisque seules des sources publiques ont été consultées afin d'établir le portrait " public » de la GC au Québec. Les exemples présentés ont pour but d'illustrer les types de gestion des connaissance en cours au Québec tout en posant la question de son avenir.
Tous droits réservés (c) Association pour l'avancement des sciences et des techniques de la documentation (ASTED) et Corporation des bibliothécaires professionnels du Québec (CBPQ), 2008
Ce document est protégé par la loi sur le droit d'auteur. L'utilisation des services d'Érudit (y compris la reproduction) est assujettie à sa politique d'utilisation que vous pouvez consulter en ligne.

https://apropos.erudit.org/fr/usagers/politique-dutilisation/ 


\title{
La gestion des connaissances dans les organisations québécoises : état des lieux, enjeux actuels, avenir
}

\author{
KIMIZ DALKIR \\ Professeure adjointe \\ École des sciences de l'information \\ Université McGill \\ kimiz.dalkir@mcgill.ca
}

\section{RÉSUmÉ | ABstracts | RESUMEN}

L'auteure passe en revue la gestion des connaissances (GC) au. Québec dans trois secteurs primaires : les grandes universités québécoises, le secteur public provincial et certaines grandes organisations du secteur privé. Une bonne partie des projets de GC implique un partenariat entre ces trois secteurs. Cependant, la GC au Québec souffre encore d'un manque de visibilité nationale et internationale. L'article ne propose pas un inventaire exhaustif puisque seules des sources publiques ont été consultées afin d'établir le portrait "public" de la GC au Québec. Les exemples présentés ont pour but d'illustrer les types de gestion des connaissance en cours au Québec tout en posant la question de son avenir.

Knowledge Management in Quebec Organizations : State of Affairs, Current Issues, Future

The author reviews knowledge management (KM) in Quebec in three primary areas : major universities, the public sector, and certain large private sector organizations. A large number of KM projects involve partnerships between these three sectors, however, $K M$ in Quebec suffers from a lack of national and international visibility. This article does not present an exhaustive list of projects since only public sources were consulted to establish a "public" portrait of KM in Quebec. Examples are presented as case studies of knowledge management in progress in the province while considering its future.

\section{La gestión del conocimiento en las organizaciones quebequenses : estado actual, retos y perspectivas para el futuro}

La autora analiza las prácticas de gestión del conocimiento (GC, o KM por sus siglas en inglés) en Québec en tres sectores primiordiales: las grandes universidades quebequenses, el sector público provincial y algunas grandes organizaciones del sector privado. Una gran parte de los proyectos de GC implica la colaboración entre estos tres sectores. Sin embargo, la GC en Québec sufre de una gran falta de visibilidad nacional e internacional. El présente artículo no propone un inventario exhaustivo, ya que solo fueron consultadas las fuentes públicas, para hacer el retrato "público" de la CG en Québec. Los ejemplos recopilados buscan ilustrar los tipos de GC actualmente vigentes en la provincia al tiempo que nos permiten interrogarnos sobre su devenir.
L A MAJORITÉ DES EMPLOIS de l'économie du savoir d'aujourd'hui entraîne la création et le traitement de connaissances qui, à leur tour, fournissent la clé de l'avantage concurrentiel et du succès des organisations (Marquardt, 2002). Bien gérer ce savoir devient alors une préoccupation stratégique essentielle. La gestion des connaissances (GC) est constituée de processus systématiques d'acquisition, d'organisation, de maintien, d'application, de partage et de renouvellement, à la fois implicites et explicites, du savoir des employês, dans le but d'augmenter le rendement organisationnel et de créer de la valeur (Wiig, 1993 ; Davenport et Prusak, 1998; Allee, 1997 ; Alavi et Leidner, 2001; Al-Hawamdeh, 2003; Dalkir, 2005; Choo, 2006). Les initiatives efficaces de GC aident les organisations à canaliser les connaissances ayant une valeur et une utilité pertinentes, ainsi qu'à garantir leur utilisation et leur réutilisation afin d'éviter d'avoir à réinventer la roue. Les avantages de la GC peuvent être marqués par un rendement amélioré, des économies, une position concurrentielle avantageuse et un apprentissage organisationnel efficace (Lesser et Prusak, 2004 ; Argote et Ingram, 2000).

\section{La gestion des connaissances au Québec}

La gestion des connaissances au Québec est aussi répandue dans le secteur privé (par exemple Bombardier et Hydro-Québec) que dans le secteur public. Sont présentés ci-dessous quelques exemples du genre de travail actuellement en cours en GC au Québec. Les lecteurs sont invités à consulter les références indiquées afin de se familiariser davantage avec ce sujet.

\section{Les 'universités québécoises}

Toutes les universités québécoises sont de plus en plus concernées par la GC - que ce soit par les cours offerts ou par les projets de recherche. Dans la plupart des cas, la GC est intégrée à l'un des départements suivants : la gestion, l'information ou l'éducation. TéléUniversité (conjointement avec son centre de recherche, LICEF) et l'Université Concordia ont intégré la gestion 


\section{Les initiatives efficaces de GC aident les organisations à canaliser les connaissances ayant une valeur et une utilité pertinentes, ainsi qu'à garantir leur utilisation et leur réutilisation afin d'éviter d'avoir à réinventer la roue.}

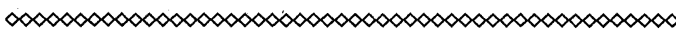

des connaissances aux technologies de l'éducation, surtout avec la recherche sur l'apprentissage en ligne (par exemple Henri et al., 2006 ; Driscoll et Carliner, 2005). L'Université Laval a entrepris plusieurs études importantes sur la gestion des connaissances et compte des étudiants au doctorat dans ce domaine (par exemple Ouimet et al., 2006). L'Université de Montréal, par l'entremise des Hautes études commerciales (HÉC) et de l'École de bibliothéconomie et des sciences de l'information (EBSI), a participé à de nombreuses initiatives importantes en matière de GC (par exemple Dubé et al., 2006).

L'UQAM compte plusieurs départements qui effectuent des recherches sur la GC (pour un exemple appliqué au réseau forestier, voir Mankai et al., 2003). Finalement, à l'École des sciences de l'information de l'Université McGill, plusieurs études sur la GC sont en cours en partenariat avec l'EBSI (par exemple Bergeron, 2000) de l'Université de Montréal, dans le domaine de la veille concurrentielle et de la GC (par exemple Bouthillier et Dalkir, 2005), et avec le CEFRIO (Centre francophone d'informatisation des organisations) pour aider Oxfam Québec à devenir une organisation apprenante.

La province de Québec a développé une approche réseau en santé et sécurité au travail qui est très estimée par les praticiens et les chercheurs dans ce domaine à travers le Canada. Son centre de recherche, l'Institut de recherche Robert-Sauvé en santé et en sécurité du travail (IRSST), privilégie également une approche réseau. En mars 2001, les Instituts de recherche en santé du Canada (IRSC) ont formé une équipe d'" alliance communautaire pour la recherche en santé » basée à l'Université Memorial (SafetyNet).

\section{Le gouvernement du Québec}

Le Québec a profité de l'existence d'un organisme unique; le CEFRIO, qui a aidé à promouvoir les recherches sur la GC ainsi qu'à l'implanter dans plusieurs organisations des secteurs privé et public. Le CEFRIO ${ }^{1}$ est un organisme qui compte plus de 100 membres universitaires, gouvernementaux et de l'industrie ${ }^{2}$, de même que plus de 50 chercheurs associés. Il aide les organisa-

1. http://www.cefrio.q̨c.ca/cefrio.cfm

2. Voir http://www.cefrio.quc.ca/dev_lis.cfm pour une liste complette. tions à mieux atteindre leurs objectifs de rendement et d'innovation en réunissant et en soutenant les meilleurs chercheurs et praticiens. Les organisations sont d'abord inventoriées de manière à circonscrire leurs besoins ; des projets de recherche sont ensuite développés pour fournir un banc d'essai aux chercheurs. Certaines recherches du CEFRIO sur la GC comprennent la transmission intergénérationnelle du savoir-faire, les outils de collaboration pour de nouvelles façons de travailler, les communautés de pratique pour l'apprentissage, de même que des études d'ensemble et des analyses comparatives. Les résultats des recherches sont ensuite communiqués à tous les membres.

Parmi les projets de recherche en gestion du savoir, on retrouve :

- Comment repérer, préserver et partager les connaissances stratégiques auprès de son personnel, particulièrement lors du départ d'un employé expérimenté ? (Ermine et al., 2006)

- Comment analyser les pratiques exemplaires en gestion des connaissances de quatre entreprises québécoises : Bell, Banque Nationale, CSST et DMRÉ ? (Jacob, 2000)

- Comment utiliser les nombreuses formes de partage de connaissances et de savoirs à la CSST ? (Benoit, 200o)

\section{Le secteur privé}

Dans le secteur privé au Québec, la plupart des initiatives de GC ont été mises en place pour améliorer l'efficacité (en augmentant la valorisation et en diminuant la répétition du travail), la collaboration et le travail d'équipe, pour promouvoir une meilleure innovation, ainsi que pour examiner la problématique des travailleurs qui prennent leur retraite. Ce dernier aspect constitue souvent le catalyseur du premier projet en GC. Au Québec, comme c'est le cas ailleurs, presque tous les genres d'entreprises et d'industries, de n'importe quel secteur d'activités et de n'importe quelle taille, sont généralement impliqués dans des activités de GC. Citons par exemple Hydro-Québec, qui s'est penchée sur les ressources humaines et la GC pour son plan de relève, des compagnies d'assurances et des banques telles que Standard Life, Clarica et la Banque de Montréal, qui tentent de promouvoir un meilleur partage des connaissances à travers leur organisation, ainsi que les secteurs forestier et manufacturier qui se sont centrés sur l'organisation des connaissances stratégiques pour un meilleur accès et une meilleure récupération. Quelques exemples de projets sont présentés ci-après.

Une étude portant sur 163 compagnies manufacturières du Québec (dont près de $90 \%$ sont des petites et moyennes entreprises) a été réalisée afin d'examiner les méthodes de gestion des connaissances (Halley et Beaulieu, 2005). L'étude a démontré que l'implantation de la GC entraînait une meilleure conception des 
connaissances ayant une valeur stratégique, de même qu'une implantation de mesures destinées à protéger les connaissances jugées stratégiques.

Dans le secteur forestier du Québec, Van Horne et Marier (2004) ont utilisé un jeu en guise d'outil innovateur de gestion et de transmission des connaissances implicites et explicites des chercheurs et praticiens aux employés des entreprises. La perspective de la retraite et de la perte des connaissances de milliers de professionnels à travers le monde entre en ligne de compte dans les défis des entreprises forestières en regard des économies et de la création de la valeur. Ces défis exigent des entreprises qu'elles améliorent la gestion et la transmission de toutes les connaissances disponibles, ce qui requiert ensuite des solutions novatrices. Le jeu du bois (Quebec Wood Supply Game) soumet des problèmes de GC et propose des solutions possibles pour des activités logistiques commerciales ; par exemple, les fonctions de planification, d'approvisionnement et de collaboration du réseau de création de valeur de l'industrie des produits forestiers québécois.

Dans certains cabinets d'avocats tels que Ogilvy Renault et Stikeman Elliott, des employés se consacrent à la GC et intègrent des technologies de collaboration comme des wikis, de façon à mieux partager leur savoirfaire, à accélérer le rythme d'apprentissage et de formation, ainsi qu'à mieux organiser leurs connaissances.

Le Cirque du Soleil compte à la fois un directeur de la gestion des connaissances et une vice-présidente de la gestion des connaissances et des technologies de l'information qui est également responsable de la gestion des connaissances de l'entreprise. À ce titre, elle est responsable de canaliser et de gérer les connaissances reliées à la créativité impliquée dans la conception de spectacles, de même que les connaissances associées à la gestion de l'entreprise elle-même.

Il existe plusieurs entreprises de technologies de la GC au Québec. L'une d'entre elles, Nstein, a récemment été choisie comme "produit phare de 2007 " par les rédacteurs de la revue KM World ${ }^{3}$. Nstein créé une suite logicielle de gestion de contenu qui est utilisée dans plusieurs applications de GC. En 2004, un groupe informel appelé Agora a été créé afin d'aider les entreprises à réussir et à partager leurs expériences de GC. Les membres comprennent entre autres Hydro-Québec, Bell, le Mouvement des Caisses Desjardins, Alcan et IBM (Bromont).

\section{Conclusions}

La gestion des connaissances (GC) est de plus en plus intégrée dans une gamme variée d'organisations à travers le monde, au Canada et au Québec. Les professionnels des bibliothèques et de l'information ont des rôles déterminants à jouer dans le développement et le maintien des grands systèmes de GC puisque de

3. http://www.nstein.com/en/awards.php.
Dans le secteur forestier du Québec, Van Horne et Marier (2004) ont utilisé un jeu en guise d'outil innovateur de gestion et de transmission des connaissances implicites et explicites des chercheurs et praticiens aux employés des entreprises.

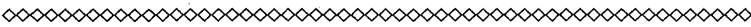

nouveaux rôles et de nouvelles responsabilités, tant du côté pratique (nouveaux rôles professionnels) que théorique (nouveaux cours et nouvelles formations), sont créés. La gestion des connaissances prendra de plus. en plus d'importance puisque les nouvelles méthodes de travail demandent une meilleure collaboration, de l'interaction virtuelle, de nouvelles structures d'organisation telles que des communautés de praticiens. qui rassemblent des professionnels de mệme sensibilité et, évidemment, un besoin de pérennité du savoir. La pérennité du savoir, dans le sillon de la continuité des affaires, renvoie aux initiatives de GC qui doivent être mises en place pour s'assurer que les connaissances essentielles (à la fois implicites et explicites) ne disparaissent pas à causé de la rotation, de la retraite ou de l'attrition des employés. Les -connaissances attestées nécessiteront toujours une interprétation (ou du moins des gens sachant où ces connaissances ont été classées). Les connaissances implicites seront perdues lorsque les gens quitteront l'organisation, ce qui risque d'entraîner une " amnésie d'entreprise » (Kransdorff et Williams, 2000).

En 2003, le premier ministre de la France a déclaré que le Canada ainsi que le Québec possédaient tous les ingrédients essentiels à une économie du savoir. Montréal, en particulier, était célèbre pour avoir un quotient élevé de connaissances et d'innovation. David Florida (Stolarick et Florida, 2006) a également classé Montréal au nombre des cinq grandes villes du savoir au monde - avec un énorme potentiel de "capital créatif ». Dans son étude de neuf mois sur Montréal, il a estimé que :

Plus de la moitié de la population est bilingue et près d'un quart parle trois langues; avec quatre grandes universités, elle a presque autant d'étudiants par habitant que Boston; et elle abrite un ensemble florissant de projets artistiques et culturels qui reçoivent un soutien public (par exemple le Fonds de la musique du Canada du gouvernement fédéral, le crédit d'impôt accordé aux lieux artistiques et la Société de développement des entreprises culturelles du Québec). Cela peut ressembler au genre de propos qu'une chambre de commerce publierait, mais vous devez admettre que Montréal fait quelque chose de bien. Avec la croissance rapide de l'emploi des dernières années, la 


\section{En 2003, le premier ministre de la France a déclaré que le Canada et le Québec possédaient tous les ingrédients essentiels à une économie du savoir.}

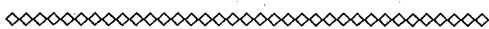

ville a attiré plusieurs entreprises importantes, incluant l'entreprise française de jeux vidéo Ubisoft.

(De Wolf, 2005)

La gestion des connaissances est donc bien vivante au Québec, même si elle demeure en quelque sorte un secret bien gardé. La gestion des connaissances a fait l'objet de beaucoup de publications et de conférences, de sorte qu'il peut être difficile de 's'y retrouver. Un leadership en gestion des connaissances est nécessaire pour aider les chercheurs, enseignants et praticiens de la GC à « exercer ce qu'ils prêchent ». La GC du Québec doit se faire davantage en communauté afin d'accroître les occasions de collaboration et de partage de ce qui a plus ou moins bien fonctionné. Les universités, les gouvernements et les entreprises doivent travailler ensemble afin de promouvoir, d'implanter et de mieux faire connaître la gestion des connaissances au Québec. $\uparrow$

\section{Sources consultées}

Alavi, M. and D. Leidner, 2001. Review : knowledge management and knowledge

management systems : conceptual foundations and research issues. MIS Quarterly, 23(1) : 107-136.

Al-Hawamdeh, S. 2003. Knowledge management. Oxford, UK: Chandos Publishing.

Allee, V. 1997. The Knowledge Evolution: Expanding Organizational Intelligence. New York: Butterworth-Heinemann.

Argote, L. and P. Ingram. 2000. Knowledge transfer : a basis for competitive advantage in

firms. Organizational Behavior and Human Decision Processes, 82(1) : 150-169.

Benoit, J. 2000. Une communauté de pratique élargie : le forum et la base de connaissances des inspecteurs de la Commission de la santé et de la sécurité du travail (CSST) 1996-1999.

CEFRIO : Collection recherches et études de cas, avril 2000, $76 \mathrm{p}$.

Bergeron, Pierrette. 2000. Veille stratégique et PME. Comparaison des politiques gouvernementales de soutien. Sainte-Foy: Presses de l'Université du Québec, 462 p. (Collection : gestion de l'information).

Bouthillier, F. and K. Dalkir. 2005. Knowledge management and competitive intelligence : examination of similarities, differences and intersections. S. Hawamdeh (ed.). Knowledge management: Nurturing culture innovation and technology. Proceedings of the 2005 International Conference on Knowledge Management. New Jersey: World Scientific, 603-610.

Choo, C. 2006. The Knowing Organization: How organizations use information to construct meaning, create knowledge and make decisions. Second Edition. New York: Oxford University Press.

Dalkir, K. 2005. Knowledge management theory and practice. Boston, MA : Elsevier, Butterworth-Heinemann.
Davenport, T. and L. Prusak. 1998. Working knowledge : how organizations manage what they know. Boston, MA : Harvard Business School Press.

De Wolf, C. 2005. Creative class war : the debate over Richard Florida's ideas. Disponible en ligne à : p. (Consulté sur Internet le 30 novembre 2007).

Driscoll, M. and Carliner, S. 2005. Advanced web-based training: Adapting real world strategies in your online learning. San Francisco : Pfeiffer.

Dubé, L., Bourhis, A. and R. Jacob. 2006. Towards a Typology of Virtual Communities of Practice. Interdisciplinary Journal of Knowledge Management, $1: 69-93$.

Ermine J-L, Boughzala, I. and T. Tounkara. 2006. Critical knowledge map as a decision tool for knowledge transfer actions. The Electronic Journal of Knowledge Management, 4(2) :129-140.

Halley, A. and M. Beaulieu. 2005. Knowledge management practices in the context of supply chain integration : the Canadian experience. Supply Chain Forum, 6(1). Disponible enligne à : http://www.supplychain-forum.com/. (Consulté sur Internet le 30 novembre 2007).

Henri, F., Gagné, P., Maina, M., Gargouri, Y, Bourdeau, J. and G. Paquette. 2006. Development of a knowledge base as a tool for contextualized learning, in AI \& SOC, August 2005. London: Springer-Verlag.

Jacob, R. 2006. Gérer les connaissances : un défi de la nouvelle économie du $21^{\mathrm{e}}$ siècle. Réal Jacob. Collection recherches et études de cas, CEFRIO. $61 \mathrm{p}$.

Kransdorff, A. and R. Williams. 2000: Managing organizational memory: the new competitive imperative. Organizational Development Journal, 18(1) : 107-117.

Lesser, E. and L. Prusak. 2004. Creating value with knowledge. Oxford, UK: Oxford University Press.

Mankai, A., Lejeune, A. et S. Lebel. 2003. Étude de cas : une initiative de gestion des connaissances dans le contexte de la fonction publique au Québec : le processus de création et d'exploitation des fiches de dossiers stratégiques au ministère des Transports du Québec. Les actes du colloque, XII Conférence de l'Association Internationale de Management Stratégique. Disponible en ligne au : http://www.strategie-aims.com/tunis/ . resumes/RSP_2ob.pdf. (Consulté sur Internet le 30 novembre 2007)

Marquardt, M. 2002. Building the Learning Organization. $2^{\text {nd }} \mathrm{ed}$. Palo Alto, CA : Davies-Black Publishing, Inc.

Ouimet, M., Landry, R., Amara, N. and O. Belkhodja. 2006. What Factors Induce University Researchers to Transfer their Research Knowledge to Users Outside the Scholarly Community ? Evidence from Researchers in Canadian Medical Schools. Social Science and Medicine, 62(4) : 964-976.

Roy, M., Parent, R. and L. Desmarais. 2003. Knowledge Networking : A Strategy to Improve Workplace Health \& Safety Knowledge Transfer. Electronic Journal of Knowledge Management (EJKM), 1(2). Disponible en ligne à : http:// www.ejkm.com/volume-1/volume1-issue-2/v1-i2-articles.htm. (Consulté sur Internet le 30 novembre 2007)

Stolarick, K. and D. Florida. 2006. Creativity, connections and innovation : a study of linkages in the Montreal region. Environment and Planning, 38(10) : 1799-1817.

Van Horne, C. and P. Marier. 2004. The Québec wood supply game: an innovative tool for knowledge management and transfer. Disponible en ligne à : http://forac.fsg.ulaval.ca/fileadmin/ docs/Publications/QCWoodSupplyGame.pdf. (Consulté sur Internet le 30 novembre 2007)

Wiig, K. 1993. Knowledge Management Foundations, vol 1. Arlington TX : Schema Press. 